\title{
TENSILE BOND STRENGTH OF SELF-ADHERING FLOWABLE COMPOSITE ON DENTIN SURFACE (AN IN VITRO STUDY)
}

\author{
Ahmed A Abdul Aziz ${ }^{*}$, Nady I brahim**, Ahmed Tharwat***
}

\begin{abstract}
This study evaluated the effect application microtensile bond strength ( $\mu \mathrm{TBS}$ ) on the self-adhering flowable composite resin to dentin surface. Occlusal surfaces of human adult premolars and deciduous molars were ground to obtain flat dentin surfaces with a water-cooled diamond saw. Each of the following adhesive systems were applied to dentin surface of the tooth, following manufacturers' instructions: Self-adhesive flowable resin composite: (Vertiseflow), Self-etch adhesive system (optibond). Buildups were then made on the bonded surfaces and incrementally light-cured for $20 \mathrm{~s}$. with Two light curing resin composite resin: Herculite resin composite and Self-adhesive flowable resin composite. The restored teeth were stored in water at $37^{\circ} \mathrm{C}$ for $24 \mathrm{~h}$, three months and six months. Each tooth quarter was serially cut in a longitudinal direction in order to obtain several bonded sticks (0.9-mm2 in cross-section). Maximal microtensile stress (in MPa) at failure was recorded using a universal testing machine. Data were analyzed by two-way ANOVA and Tukey's test at 5\% significance level. The result revealed that self-adhesive flowable composite showed the lowest tensile bond strength in comparison with the obti bond all-in-one adhesive or Vertise flow as adhesive. In conclusion, Optibond adhesive has higher bond strength than Vertise flow adhesive composite, etching dentin surface with acid etchant phosphoric acid 37\% enhance bond strength for self-adhesive flowable composite, Tensile bond strength in permanent dentin higher than deciduous dentin and the storage time has adverse effect on the bond strength.
\end{abstract}

\section{INTRODUCTION}

The first generation of flowable composites was introduced in 1996 to restore Class V lesions (1). The flowable composites can be easily inserted into small cavities and are expected to exhibit better adaptation to the internal cavity wall compared to the conventional restorative composites, which are more viscous ${ }^{(2)}$.

Using of flowable composites would result in less microleakage, internal voids and postoperative sensitivity ${ }^{(3)}$. Today, there are wide ranging applications for them, such as small Class III and Class V cavities, pits and fissures, a base for composite restorations, amalgam margin repairs, enamel defects and incisal edge repairs in anterior sites. In addition, recently they are being used as filled adhesives ${ }^{(4)}$.

The flowable composite materials are a modification of restorative resin composites, so they tend to contain a lower filler content (weight: 60 $70 \%$; volume: $46-65 \%$ ) than their hybrid analogs (weight:70-80\%; volume: 60-75\%) (5). Reduced filler loading of flowable composites leads to enhanced flow and reduced elastic modulus ${ }^{(6)}$.

After decades of evaluation, adhesives may include different formulations and, consequently, their bond values may vary in relation to dental substrate. Currently there is a tendency to simplify bonding procedures which introduced the selfetching adhesive concept ${ }^{(7)}$.

\footnotetext{
* Demonstrator of Operative Dentistry Faculty of Dental Medicine South valley University.

** Assistant Professor of Operative Dentistry Department. Faculty of Dental Medicine Al-Azhar University (Boys)Cairo. *** Assistant Professor and head of Operative Dentistry Department Faculty of Dental Medicine Al-Azhar University (Boys) Assuite.
} 
Recently in adhesive dentistry is the development of a self-adhering flowable composite resin. It is a direct composite resin restorative material that has an adhesive resin incorporated into the flowable composite resin ${ }^{(8)}$.

Self-adhering flowable composite resin is based on bonding technology that utilizes glycerophosphate dimethacrylate (GPDM) is a functional monomer to etch enamel and dentin which acts like a coupling agent, it also contains hydroxy ethyl methacrylate (HEMA) is another functional monomer which most commonly used in dental adhesives to enhance wetting and resin penetration in $\operatorname{dentin}^{(9)}$.

\section{MATERIAL AND METHODS}

The teeth that selected in this study, divided into (63 permanent (premolar)) and (63 deciduous (molar)). All the selected teeth were free from decay, abnormalities or restorations. The collected teeth examined by visual inspection and by using magnification lens of X7 to exclude any tooth with cracks or other structural defects. They were washed by running tap water and cleaned from any plaque, calculus or attached periodontal tissue then stored in distilled water till testing.

The permanent and deciduous tooth specimens were randomly divided into three equal groups of $(n=21)$ specimens. According to the adhesive system, as follows: group1(V): Vertise flow used as a direct restoration, group 2(VH): Vertise flow and Herculite ultra universal Nanohybrid Dental Composite (used as bonding agent). group 3(OH): OptiBond with Herculite ultra universal Nanohybrid Dental Composite.

Each group was then divided equally into three subgroups, according to different aging periods, subgroup1: (A1) $(n=7)$ aged for one day, subgroup2: (A2) $(n=7)$ aged for three months and subgroup3: (A3) $(n=7)$ aged for six months.

The occlusal surface of each tooth was ground to flat level, parallel to the occlusal surface and perpendicular to the long access of the tooth using a cylindrical diamond stones mounted in high speed hand piece accompanied with copious air-water spray

\section{Application of the restorative materials:}

All materials in this study were used according to the manufacturer's instructions.

\section{A-Self-adhesive resin composite application:}

The exposed dentin surface was rinsed and dried gently, Vertise flow was dispensed with the provided dispensing tip on the prepared dentin surface. Then, light cured for 20 seconds using LED curing unit, according to the manufacturer instructions. Then the split copper mold was placed on bonded surface and Vertise flow was injected into the mold of $2 \mathrm{~mm}$ height and light cured for 20 seconds, the distance between light output and resin composite was zero distance by using Mylar strip, used as a separating material.

\section{B-Self-etch adhesive and the resin composite:}

The exposed dentin surface was rinsed and dried lightly, OptiBond All-In-One self-adhesive system was applied with scrubbing motion for 20 seconds for all specimens by using small brush, and then air dried gently for 5 seconds and light cured for 10 seconds using LED curing unit with a light output of $1000 \mathrm{~mW} \backslash \mathrm{cm} 2$ and the distance between light output and resin composite was zero distance by using celluloid strip used as a separating material, according to the manufacturer's instructions.

The restorative material (Herculite Ultra resin composite) was carefully applied to the dentin surface by applying the material in a split copper mold. Composite was placed in one increment of $2 \mathrm{~mm}$ height and light cured for 20 seconds.

\section{C- Self-adhesive resin composite and Microhy- brid resin composite:}

The exposed dentin surface etched with $37 \%$ phosphoric acid for 15 seconds, then rinsed for 15 seconds with distilled water and dried lightly with 
absorbent pellet and air. Vertise Flow was dispensed with the provided dispensing tip on the prepared dentin surface and cured for 20 seconds. Then the Add microhybrid resin composite, which cured for 20 seconds according to the manufacturer's instructions.

The restorative material (flowable resin composite) was carefully applied to the dentin surface as mentioned before. The light intensity was regularly checked using Radiometer.

After completion of the bonding procedures, 2 $\mathrm{mm}$ horizontal increments of composite were built up to a height of $2 \mathrm{~mm}$ on the dentin surface with an approximate $2 \times 4 \mathrm{~mm}$ cross sectional area, and each increment was light cured for 20 seconds.

\section{Storage of the specimens:}

All samples were stored in distilled water at $\left(37^{\circ}\right)$ in incubator to regulate the aging temperature $\left(37^{\circ}\right)$ either for one day, three months or six months. The storage distill water was changed every week for all the aged specimens. To simulate the oral condition during storage time, the specimens were thermocycled between $5^{\circ} \mathrm{C}$ and $55^{\circ} \mathrm{C}$ for 5000 cycles (one minute for each).

\section{Specimens preparation for microtensile bond strength testing:}

Each tooth was mounted on the cutting machine and sectioned into a series of $1 \mathrm{~mm}$ thick slabs under cooling water. The sectioning was performed using a diamond disc of 4"diameter x $0.3 \mathrm{~mm}$ thickness x 0.5 " arbor impregnated diamond cutting blades with wear-resistant Ti-C coating. 4 central sticks are selected from each tooth; each stick has $1.0 \mathrm{~mm}^{2}$ cross-section checked by digital caliper.

Each specimen was attached with its ends to a specially designed, modified version of Ciucchi's jig using the cyanoacrylate adhesive (Zapit).

The applied tensile force resulted in debonding along the substrate-adhesive interface

\section{Statistical analysis:}

Data were recorded, tabulated and submitted for proper statistical analysis using Asistat 7.6 statistics software for Windows.

\section{RESULTS}

\section{Effect of restorative material on Microtensile Bond Strength ( $\mu$ TBS)}

Descriptive statistics showing mean values, standard deviations $( \pm \mathrm{SD})$ for $\mu$-tensile bond strength measured in (MPa) recorded for both Vertise flow composite groups as function of adhesive system approach, dentin type and storage time are summarized in table.

\section{Effect of type of dentin structure on micro tensile bond strength $(\mu \mathrm{TBS})$}

\section{A. Effect of adhesive system on $\mu$-tensile bond strength on permanent tooth:}

Regardless to adhesive system approach, dentin substrate or storage time, totally it was noted that (optibond+Herculite) group recorded statistically significant higher $\mu$-tensile bond strength mean \pm SD values $(28.17 \pm 2.16 \mathrm{MPa})$ than (Vertise flow + Herculite) group mean \pm SD values $(27.93 \pm 2.53$ $\mathrm{MPa}$ ), and the least result recorded by (Vertise) group $(24.35 \pm 3.04 \mathrm{MPa})$, as indicated by ANOVA test followed by pair-wise Tukey's post-hoc.

\section{B. Effect of adhesive system on $\mu$-tensile bond strength on Deciduous tooth:}

Regardless to adhesive system approach, dentin substrate or storage time, totally it was noted that (Opti bond + Herculite) group recorded statistically significant higher $\mu$-tensile bond strength Mean \pm SD values $(17.08 \pm 3.06 \mathrm{MPa})$ than (Vertise flow + Herculite) group Mean \pm SD values $(16.89 \pm 3.03 \mathrm{MPa})$ and the lowest value was for Vertise(V) group $(14.17 \pm 2.95 \mathrm{MPa})$, as indicated by ANOVA test followed by pair-wise Tukey's posthoc tests $(\mathrm{p}<0.05)$. 


\begin{tabular}{|c|c|c|c|c|c|c|}
\hline Adhesive & \multicolumn{2}{|c|}{ Vertise flow group } & \multicolumn{2}{c|}{ Verise flow + Herculite } & \multicolumn{2}{c|}{ Opti bond + Herculite } \\
\hline Time Dentin & $\mathrm{P}$ & $\mathrm{D}$ & $\mathrm{P}$ & $\mathrm{D}$ & $\mathrm{P}$ & $\mathrm{D}$ \\
\hline $24 \mathrm{hr}$. & $27.49 \pm 1.39$ & $17.11 \pm 1.02$ & $30.06 \pm 1.35$ & $20.0 \pm 3.12$ & $30.40 \pm 1.19$ & $20.14 \pm 1.17$ \\
\hline Three months & $24.16 \pm 2.72$ & $14.20 \pm 0.98$ & $27.83 \pm 1.72$ & $16.88 \pm 1.29$ & $28.03 \pm 1.52$ & $17.1 \pm 1.71$ \\
\hline Six months & $21.41 \pm 2.06$ & $11.20 \pm 1.15$ & $25.0 \pm 2.02$ & $13.81 \pm 3.07$ & $26.08 \pm 1.01$ & $14.02 \pm 2.48$ \\
\hline
\end{tabular}

$\mu$-Tensile bond strength results (Mean values $\pm S D$ ) for both Vertise flow composite group, opti bond group and

Vertise + Herculite group, according to dentin type and storage time.

\section{Effect of storage time on $\mu$-tensile bond strength $(\mu \mathrm{TBS})$ :}

Irrespective of composite type, adhesive system approach or dentin substrate, totally it was noted that $24 \mathrm{hr}$ storage time subgroup recorded statistically significant highest $\mu$-tensile bond strength mean values $(24.39 \pm 6.02 \mathrm{MPa})$ followed by three-month storage time subgroup mean values $(21.31 \pm 5.96$ $\mathrm{MPa}$ ) while six-month storage time subgroup recorded statistically significant lowest mean values $(18.22 \pm 6.27 \mathrm{MPa})$ as indicated by ANOVA test $(p<0.05)$. Pair-wise Tukey's post-hoc test showed non-significant difference between $24 \mathrm{hr}$ and threemonth storage time subgroups $(\mathrm{p}>0.05)$

\section{DISCUSSION}

The bond strength of enamel has been studied extensively, bonding to dentin with the generations of bonding systems has remained unsolved. The dentin is characterized as a biologic composite of collagen matrix filled with apatite crystals dispersed between parallel micrometer-sized hyper mineralized collagen poor dentinal tubules containing peritubular dentin. Bond strength testing is relatively easy, fast and remains most popular methodology for measuring the bonding effectiveness of adhesive systems. Most authors agree that measuring the microtensile bond strength is a fundamental importance to evaluate the bonding strength ${ }^{(10)}$.

\section{I) Discussion of materials \& methods:}

In this study every effort was exerted to standardize the methodology and to mimic the clinical conditions as close as possible. In an attempt to standardize dentin substrate only used extracted sound deciduous molars were chosen to offer greater occlusal surface for application of adhesive and resin composite, Also the premolars were selected in permanent teeth to get sound extracted teeth for orthodontic treatment reasons.

In the present study, flat dentin surfaces were used as bonding substrates, which are not subjected to the same polymerization contraction stresses faced on three-dimensional tooth preparations ${ }^{(11)}$.

\section{1- Selection of adhesive systems:}

Self-adhesive flowable resin composite (Vertise Flow) represents a new generation in flowable composite technology by effectively fusing together self-adhesive and restorative technology in one product. Its self-adhesive property allows it to bond to enamel and dentin without etching, rinsing, priming or bonding. Consequently, the application procedures are simplified, shortened and made easier. In addition, its mild self-etch property offers the potential for reduced sensitivity ${ }^{(12)}$

Self-adhering flowable composite resin is based on bonding technology that utilizes glycerophosphate dimethacrylate (GPDM), a functional monomer, to etch enamel and dentin. This resin 
bonds in two ways: Primary through the chemical bond between the phosphate functional groups of a GPDM monomer and calcium ions of the tooth.

Secondary, through a micromechanical bond as a result of an inter-penetrating network formed between the polymerized monomers of selfadhering flowable composite resin and collagen fibers (as well as the smear layer) of dentin ${ }^{(12)}$

OptiBond All-in-One as one-step self-etch adhesive system was used in this study because it has the same adhesive technology which incorporated into the Vertise Flow composite.

\section{2-Light cure unit:}

In the present study, LED curing unit was used to overcome the decrease of light intensity of the halogen light curing units occurs over time due to bulb and filter aging ${ }^{(13)}$. Also, LEDs have a working lifetime of over $10,000 \mathrm{~h}$, compared to $40-100 \mathrm{~h}$ for halogen bulb ${ }^{(14)}$, and wavelength peaks around $470 \mathrm{~nm}$, LED which is nearly similar to the most commonly used photo-initiator camphor quinone (CQ) in dental composites so negating the need for filters. Furthermore, the thermal emission of the LED light curing units is significantly lower than that of halogen light curing units. Also, the degree of conversion and depth of cure of LEDs were higher compared to than halogen light curing unit ${ }^{(15)}$.

\section{3 -Selection of deciduous and permanent dentin :}

Comparing between permanent and deciduous dentin to differentiate between the two-dentin type according to physiological differences. The dentinal tubules are more regular and highly mineralized in permanent dentin and absence of interglubular dentin in deciduous dentin, which affect in bonding strength and durability of restorative materials ${ }^{(16)}$.

\section{4- Water storage, Thermocycling and tensile bond strength test:}

The most commonly used artificial aging technique is long-term water storage at $37 \mathrm{C}$ for a specific period (De Munch et al 2005). So distilled water was used in this study as storage media for the bonded specimens, for 24 hours, 3 months and 6 months ${ }^{(17)}$.

In this study, specimens were subjected to thermocycling to closely simulate the thermal changes in the oral cavity. These changes may accelerate hydrolysis of interface components and subsequent uptake of water and extraction of breakdown products or poorly polymerized resin monomers ${ }^{(18)}$.

Durability of dentin bonding can be affected by many factors such as: time, temperature chemical and mechanical interactions. Several comparative studies have been published to evaluate the changes of dentin bonding strength. These studies compared bond strength values between different types of dentin bonding systems at different times and under various changing factors. Different artificial aging techniques were used to resemble the changes in oral cavity. The most commonly used method is water storage, where the bonded specimens are stored in $37^{\circ} \mathrm{C}$ water for a certain time ${ }^{(19)}$.

\section{II) Discussion of results:}

\section{A. Effect of different adhesive systems on tensile bond strength:}

Data revealed that self-adhesive flowable composite showed the lowest tensile bond strength in comparison with the obti bond all-in-one adhesive or Vertise flow as adhesive. This may be due to bonding mechanism relies on the adhesive monomer glycerol phosphate dimethacrylate (GPDM). Specifically, the phosphate group of GPDM is responsible for acid etching. The dimethacrylate functional groups are involved in cross-linking reactions with other methacrylate monomers, thus providing mechanical strength to the adhesive material. Based on the $\mathrm{pH}$ declared form the manufacturer (1.9), Vertise Flow can be expected to interact with dental substrate similarly to a mild self-etch adhesive not as the total etch adhesive ${ }^{(20)}$. Also, the self-adhering flowable 
resin composite Vertise Flow is more viscous, does not contain solvent and has lower wettability. These properties could represent a drawback for the material's ability to wet self-etched collagen fibrils ${ }^{(21)}$.

This study antagonist with Merve E. et al. who examined the shear bond strength of Vertise Flow to dentin along with other self-etch adhesive systems. They reported that the shear bond strength of Vertise Flow was lower than that of Optibond All-InOne; however, this difference was not statistically significant ${ }^{(22)}$.

The data revealed that the using Vertise flow as adhesive system with two steps (etch and rinse) have better tensile bond strength through the storage time. Probably, the little performance of self-etching adhesive and self-adhering flowable resin composite is related to the little micromechanical interaction of their resin components with dentin.

The presence of a functional copolymer of methacrylate of polyacrylic and polyalkenoic acids in the conventional adhesive systems increases their resistance to the harmful effect of humidity in an environment with high relative humidity and satisfactory amount of resin component in these adhesives ${ }^{(23)}$.

Also agreed with André P. et al in 2013, who found that is very simple to use self-adhering flowable resin composite without a separate adhesive, would bond less effectively to dentin than when a resin composite would be bonded to dentin using the multi-step three step etch $\&$ rinse adhesive. He claimed that it may be due to the functional monomer used in Vertise Flow which is glycerol phosphate dimethacrylate (GPDM) ${ }^{(24)}$.

\section{B. Effect of storage time on tensile bond strength:}

The results of this study revealed that all the adhesive systems have relative best bond strength through the 24 hours. The better tensile bond strength through this period of water storage may be due to the short time that lapse of water storage or may be due to strength of the adhesive system itself through this period and strong hybrid layer therefore, may resist polymerization depending stresses.

Also, in this study showed that tensile bond strength of all the adhesive systems decreased after three months of storage time. This might be due to hydrolytic degradation of the resin and collagen fibers in the submicron spaces of the hybrid layer increase with increased exposure to water. In fact, during long-term water storage, the resin absorbs significant amount of water and consequently swelling of the resin may result in the closure of any space between the bonding resin and dentin surface ${ }^{(25)}$.

The optibond adhesive systems gave the better results of tensile bond strength then self-adhering flowable resin composite after three months and six months. This might be attributed to the fact that; stronger acid may be required to dissolve the mineral phase of dentin in order to obtain sufficient resin infiltration for better adaptation and bonding to tooth structure. Also, the self-etch adhesive systems and self-adhering flowable resin composite are less acidic and did not remove the smear layer and the self-adhering flowable resin composite did not reveal any signs of micro-mechanical attachment ${ }^{(26)}$.

The structure of adhesive monomers contains one or more polymerizable groups and additional functional groups, which are linked by a custommade spacer group. The design of the spacer group influences hydrophilicity of the resulting polymer ${ }^{(27)}$. Therefore, the hydrophilic acidic phosphate group and the short spacer group in GPDM could be major factors, if not the only ones, causing the significant difference of water sorption between Vertise flow and the other resin composites.

\section{Effect of type of dentin structure on micro ten- sile bond strength}

The data revealed that the using adhesive system with permanent dentin have higher tensile bond strength through the storage time than deciduous dentin. 
The intertubular dentin plays an important role during hybrid layer formation in superficial dentin, and the contribution to resin retention is proportional to the intertubular dentin available for bonding. ${ }^{(28)}$ Theoretically, the bond strength of dentin-bonding agents at permanent or deciduous dentin is dependent on the area occupied by resin tags at the area of intertubular dentin that is infiltrated by the resin and the area of surface adhesion ${ }^{(29)}$.

\section{CONCLUSION}

Within the limitation of this study the following conclusions might be drawn:

1. Optibond adhesive has higher bond strength than Vertise flow adhesive composite.

2. Etching dentin surface with acid etchant phosphoric acid 37\% enhance bond strength for self-adhesive flowable composite.

3. Tensile bond strength in permanent dentin higher than deciduous dentin.

4. The storage time has adverse effect on the bond strength.

\section{REFERENCES}

1. Moszner N, Salz U, Zimmermann J. Chemical aspects of self-etching enamel-dentin adhesives. Dent mater. 2005; 21: 895-910.

2. Altunsoy M, Botsali MS, Sari T, Onat H. Effect of different surface treatments on the microtensile bond strength of two self-adhesive flowable composites. Lasers Med Sci 2015; 30: 1667-1673

3. Tabassian M and Culbreath T. Flow characteristics and film thick-ness of flowable resin composites. Oper Dent. 2002; 27: 248-253.

4. Frankenberger R, Lopes M, Perdigao J. Use of flowable composites as filled adhesives. Dent mater. 2002; 18: 227-238

5. Kleverlaan $\mathrm{C}$ and Feilzer A. Polymerization shrinkage and contraction stress of dental resin composites. Dent mater. 2005; 21: 1150-1157.
6. Hervas A, Martinez M, Cabanes J, Barjau A, Fosgalve P. A review of the materials and clinical indications. Med Oral Patol J. 2006; 11: 215-220.

7. Watanabe I and Nakabayashi N. Bonding durability of photo-cured Phenyl-P in TEGDMA to smear layer retained bovine dentin. Quint Int J .1993 ; 24: 335-342.

8. Cramer N, Stansbury J, Bowman C. Recent advances and developments in composite dental restorative materials. Dent Res. 2011; 90: 402-416.

9. Bowen and Phillip. Past, present and future composite resin systems . Dent clin North .1981; 25: 205-209.

10. Pegado R, Do Amaral F, Florio F, Basting R. Effect of different bonding strategies on adhesion to deep and superficial permanent dentin. Eur J Dent. 2010; 4: 110-117.

11. Pradeep K. and Nageswar R. Three-dimensional finite element analysis of stress distribution in a tooth restored with metal and fiber posts of varying diameters. Conserv Dent J. 2015; 18: 100-104.

12. Marcelo G, Patrícia M, Ana P, Paulo M, Bruna M, Toru N. Self-Etch Adhesive Systems: A Literature Review. Braz Dent J. 2015; 26: 3-10

13. Nurcan O, Yusuf Z, Funda B , Aysel G. The effect of light curing units, curing time, and veneering materials on resin cement microhardness. Dent. Sci J.2013; 8:141-146.

14. Ruggeberg W, Uhl A, Jandt K. Optical power outputs, spectra and dental composite depths of cure, obtained with blue light emitting diode (LED) and halogen light curing units (LCUs). Brit Dent J 2002;19: 459-463.

15. Marília R, Sergei G, Fernandes R, Marcelo F. Evaluation of degree of conversion and hardness of dental composites photo-activated with different light guide tips. Eur Dent J. 2013; 7: 86-93.

16. Michel G, Askok B. Kulkarni, Marian Y, Adele B. Dentin: Structure, Composition and Mineralization . Front Biosci J . 2011; 3: 711-735.

17. De Munck J, Van Landuyt K, Peumans M, Poitevin A, Lambrechts P, Braem M, et al. A critical review of the durability of adhesion to tooth tissue. J Dent Res. 2005; 84: 118-132.

18. Luciana T, Nathalie P, Alessandra R, Rosa H, Rafael Y, Julio M. Tensile bond strength of adhesive systems effect of primer and thermocycling. Pesqui Odont Bras.2002; 16: $37-42$.

19. Hussien A, Mohamed K, abeer D, ali E. The effect of water storage on micro-shear bond strength of contemporary 
composite resins using different dentin adhesive systems. Tant Dent J. 2014; 11 :47-55.

20. Mustafa A, Mehmet T, Evren O, Ebru K . Shear Bond Strength of a Self-adhering Flowable Composite and a Flowable Base Composite to Mineral Trioxide Aggregate, Calcium-enriched Mixture Cement and Biodentine. Endo J. 2015; 41: 1691-1695

21. Miyazaki, Alessandro V, Mariam M, Cecilia G, Federica P, Marco F. Bonding and sealing ability of a new self-adhering flowable composite resin in class I restorations. Clin Oral Invest .2013; 17: 1497-1506.

22. Merve E, Aylin A, Iş11 Şaroğlu S, Deniz S. Comparison of shear bond strength of self-adhering flowable composite with different flowable composites to dentin. Euro Dent J. 2016; 5: 6-10.

23. Mauro S, Alexandra P, Eugenio J, Gislaine C, Alessandro D, Alessandra R. Effect of the application time of phosphoric acid and self-etch adhesive systems to sclerotic dentin. Appl Oral Sci J. 2013; 21:196-202.
24. André P, Annelies V, Atsushi M, Marleen P, Bart V. Bonding effectiveness of self-adhesive composites to dentin and enamel. Dent Mat. 2013; 29: 221-230.

25. Oliveira, Ricardo A, Eliane A, Alexandre B, Rodivan B. Bond strength to dentin of total-etch and self-etch adhesive systems. Rev Gaúch Odont J. 2014; 62: 365-370.

26. Braga R, Cesar P, Gonzaga C. Tensile bond strength of filled and unfilled adhesives to dentin. Am Dent J. 2000; 13: 73-76.

27. Abbas G, Fleming G, Harrington E, Shortall A, Burke F. Cuspal movement and microleakage in premolar teeth restored with a packable composite cured in bulk or in increments. Dent J. 2003; 31:437-444.

28. Alireza S, Amir G, Yasushi S, and Junji. Effect of storage time and temperature on the properties of two self-etching systems. J dent 2007; 56:218-225.

29. Sano H, Yoshikawa T, Pereira P, and Pashley D. Longterm durability of dentin bonds made with a self-etching primer, in vivo. J. Dental Research 1999; 8: 906 - 11. 